\title{
O ENSINO EXPLORATÓRIO COMO METODOLOGIA DE ENSINO NO PROCESSO DE APRENDIZAGEM MATEMÁTICA POR MEIO DO ENSINO REMOTO
}

\author{
EXPLORATORY TEACHING AS A TEACHING METHODOLOGY IN THE \\ MATHEMATICAL LEARNING PROCESS THROUGH REMOTE TEACHING
}

\author{
José Renilson de Brito Lima ${ }^{1}$, Maria Raiane Cesar Abel ${ }^{2}$, Nayara Silva do Nascimento ${ }^{3}$
}

\begin{abstract}
RESUMO
Este trabalho tem como finalidade mostrar a importância das metodologias de ensino, especificamente, o Ensino Exploratório da Matemática, como meio de possibilitar aos discentes a construção do seu próprio conhecimento, adquirindo desta forma uma aprendizagem significativa. Por meio do ensino remoto, o uso desta metodologia para o ensino de matemática torna-se uma das principais alternativas em que é possível ao professor obter êxito em seus objetivos. A realização de aulas online, devido a necessidade de isolamento social como forma de conter a pandemia de Covid-19, despertou nos professores de modo geral um esforço pela busca de novas práticas visando a continuação das atividades pedagógicas. Deste modo, visto que a internet juntamente com outros softwares seria indispensável durante esse período, o uso das tecnologias digitais passou a ser um dos principais fatores para a execução das atividades letivas, levando aos gestores escolares a se adaptarem a este novo modelo. Os professores tiveram que buscar meios que fossem capazes de atrair a atenção dos discentes e conseguirem um bom desenvolvimento das aulas, permitindo assim amenizar os prejuízos causados pela crise sanitária à educação. Desta forma, os desafios para o professor de matemática são diversos, pois esta é uma disciplina que exige de forma considerável o uso das tecnologias manuais como quadro e pincel, instrumentos que concretizam as representações matemáticas, possibilitando aos alunos visualizarem como são realizados os processos matemáticos. Devido a situação apresentada, foi aplicada uma regência de forma remota na Escola de Ensino Médio em Tempo Integral Adauto Bezerra, localizada na região norte do Ceará, no município de Massapê em uma turma de $1^{\circ}$ ano, nesta aula realizada por meio do Google Meet foi abordado o conteúdo Sistema de Coordenadas no Plano Cartesiano. Com o auxílio do software Geogebra, a aula ocorreu de maneira dinâmica sendo possível demonstrar claramente as representações e os conceitos matemáticos presentes no conteúdo. Assim, a metodologia de Ensino Exploratório em conjunto com as tecnologias digitais mostrou-se como uma alternativa viável na obtenção de uma aprendizagem matemática significativa, visto que os estudantes conseguiram interagir e debater os raciocínios gerados sobre

\footnotetext{
${ }^{1}$ Estudante do curso de licenciatura em matemática pela Universidade Estadual Vale do Acaraú (UVA). Sítio Caetano, S/N, Zona Rural, Graça, Ceará, Brasil, 62365-000. rnlsnbrito@ gmail.com.

iDORCID iD: https://orcid.org/0000-0003-1881-7951.

${ }^{2}$ Estudante do curso de licenciatura em matemática pela Universidade Estadual Vale do Acaraú (UVA). Primeira Várzea, S/N, Zona Rural, Reriutaba, Ceará, Brasil, 62260-000. raianeabel16@ gmail.com. (DORCID iD: https://orcid.org/0000-0002-1736-7103.

${ }^{3}$ Estudante do curso de licenciatura em matemática pela Universidade Estadual Vale do Acaraú (UVA). Fazenda Cedro, 01, Zona Rural, Sobral, Ceará, Brasil,62107-000. nayarasilva1357@ gmail.com. 
o assunto desta regência, possibilitando-os a atuarem como protagonistas na construção de seu próprio conhecimento matemático.

Palavras-chave: Ensino Exploratório; Tecnologias Digitais; Ensino Remoto; Aprendizagem; Matemática.

\begin{abstract}
This paper aims to show the importance of teaching methodologies, specifically the Exploratory Teaching of Mathematics, as a means of enabling students to build their own knowledge, thus acquiring meaningful learning. Through remote teaching, the use of this methodology for teaching mathematics becomes one of the main alternatives in which it is possible for the teacher to achieve success in his goals. The use of online classes, due to the need for social isolation as a way to contain the Covid-19 pandemic, has aroused in teachers in general an effort to search for new practices aiming at the continuation of pedagogical activities. In this way, since the internet along with other software would be indispensable during this period, the use of digital technologies became one of the main factors for the execution of the teaching activities, leading school managers to adapt to this new model. Teachers had to look for ways to attract the students' attention and achieve a good development of the classes, thus allowing them to mitigate the damage caused by the sanitary crisis to education. In this way, the challenges for the mathematics teacher are many, because this is a subject that considerably requires the use of manual technologies such as blackboard and paintbrush, instruments that make mathematical representations concrete, allowing students to visualize how mathematical processes are performed. Due to the presented situation, a remote regency was applied in the Adauto Bezerra Full Time High School, located in the northern region of Ceará, in the city of Massapê, in a 1st year class. In this class, the content Coordinate System in the Cartesian Plane was approached through Google Meet. With the help of Geogebra software, the class occurred dynamically and it was possible to clearly demonstrate the representations and mathematical concepts present in the content. Thus, the Exploratory Teaching methodology in conjunction with digital technologies proved to be a viable alternative in achieving meaningful mathematical learning, since students were able to interact and discuss the reasoning generated on the subject of this regency, enabling them to act as protagonists in the construction of their own mathematical knowledge.
\end{abstract}

Keywords: Exploratory teaching; Digital Technologies; Remote Teaching; Learning; Math. 


\section{Introdução}

O presente trabalho tem como finalidade relatar uma experiência em sala de aula em que foi utilizado o Ensino Exploratório de Matemática, como metodologia de ensino que instiga e desafia os alunos a pensarem como fazer matemática, propiciando-lhes um senso crítico perante o uso desta disciplina e a forma como ela está presente em seu cotidiano. Assim, consta citar que o Ensino Exploratório tem como um de seus objetivos principais quebrar o paradigma da repetição dos passos de resolução dos conteúdos matemáticos e promover aos estudantes o protagonismo de sua aprendizagem.

Por meio do Programa Residência Pedagógica da Coordenação de Aperfeiçoamento de Pessoal de Nível Superior (CAPES), em que os autores deste trabalho fazem parte, são realizados estudos sobre diferentes metodologias de ensino, bem como a realização de regências para colocar em prática o conhecimento teórico adquirido. Este programa é dividido em três módulos, tal que no primeiro módulo a metodologia de ensino trabalhada foi o Ensino Exploratório, mediante este processo de aperfeiçoamento, notou-se como é importante para a formação dos professores de matemática conhecerem e saberem utilizar esta metodologia de ensino, para que possam proporcionar à seus alunos um meio de construir seu próprio conhecimento matemático.

Entretanto, devido a pandemia do Novo Coronavírus, as atividades escolares passaram a ser realizadas remotamente como forma de prevenção à saúde coletiva das comunidades. Deste modo, o uso das tecnologias digitais como suporte para o ensino e aprendizagem dos alunos, que é considerada uma prática inovadora e desafiadora para uma grande porcentagem dos docentes, passou a ser a principal alternativa para manter a área da educação em exercício. Devido a esse acontecimento os docentes que não tinham familiaridade com essa prática tiveram que modificar sua forma de ensino e ajustar-se a este novo cenário que a educação foi inserida precocemente.

Desta forma, percebe-se a importância do uso das tecnologias digitais na educação como um meio de comunicação entre aluno-aluno e aluno-professor na busca da construção da aprendizagem, principalmente na disciplina de matemática em que é necessário o professor buscar artifícios em sua didática que ajudem os discentes a compreenderem os conteúdos matemáticos. Consequentemente, o desenvolvimento da regência aplicada com foco na perspectiva de Ensino Exploratório sobre Sistemas de 
Coordenadas no Plano Cartesiano, realizada no $1^{\circ}$ ano A da Escola de Ensino Médio em Tempo Integral (E.E.M.T.I.) Adauto Bezerra, localizada na cidade de Massapê-CE, se deu seguindo estes aspectos teóricos, com o in tuito de praticar e observar o desempenho dessa abordagem de ensino no período remoto, como forma de possibilitar aos estudantes a busca por uma aprendizagem significativa .

\section{Ensino exploratório}

No processo de ensino-aprendizagem de matemática existem diversas metodologias que auxiliam na prática docente e são importantes ferramentas que possibilitam aos estudantes obter e desenvolver os conhecimentos matemáticos presentes nos conteúdos apresentados pelos professores. A priori, o ensino tradicional era comumente visto nas aulas de matemática, entretanto notou-se que esta prática de repetição gradativa de conteúdos não viabiliza aos alunos explorar os conteúdos matemáticos, e sim já recebê-los de forma pronta, influenciando-os na maioria das vezes a decorar para obter resultados positivos em exames internos e principalmente externos, como por exemplo: Sistema de Avaliação da Educação Básica (SAEB), Exame Nacional do Ensino Médio (Enem) e no caso do estado do Ceará, Sistema Permanente de Avaliação da Educação Básica do Ceará (SPAECE), entre outras avaliações. Assim, percebeu-se a importância de transformar o ensino de matemática com o intuito de uma aprendizagem significativa para os discentes. Ponte (2005, p.12) distingue “[ [...] duas estratégias básicas no ensino da Matemática - o 'ensino directo' e o 'ensino-aprendizagem exploratório'."

No ensino directo, o docente assume um papel principal para a aprendizagem dos alunos e sua didática é sistemática, ou seja, ele explica os conteúdos e comenta sobre estes, logo em seguida, o professor aplica exercícios que estimulam os alunos a repetirem os conceitos e técnicas apresentados anteriormente (PONTE, 2005). Assim, no ensino directo, a aprendizagem do estudante resume-se em assistir as aulas e na aplicação de conceitos e técnicas pré-estabelecidos, tornando o aluno um agente passivo em sua aprendizagem. Já no ensino exploratório, o estudante é o protagonista de sua aprendizagem e um agente ativo na construção dos conceitos matemáticos. Sobre o ensino exploratório, Ponte (2005) afirma que: 
A sua característica principal é que o professor não procura explicar tudo, mas deixa uma parte importante do trabalho de descoberta e de construção do conhecimento para os alunos realizarem. A ênfase desloca-se da actividade "ensino" para a actividade mais complexa "ensino-aprendizagem" (PONTE, 2005, p.13).

Dessa maneira, o ensino exploratório possibilita uma maior interação entre alunoprofessor e aluno-aluno, visto que nessa metodologia o diálogo entre os indivíduos é um dos principais precursores da formalização do conhecimento. Diante disso, de acordo com Canavarro (2011, p. 11):

\begin{abstract}
O ensino exploratório da Matemática defende que os alunos aprendem a partir do trabalho sério que realizam com tarefas valiosas que fazem emergir a necessidade ou vantagem das ideias matemáticas que são sistematizadas em discussão colectiva. Os alunos têm a possibilidade de ver os conhecimentos e procedimentos matemáticos surgir com significado e, simultaneamente, de desenvolver capacidades matemáticas como a resolução de problemas, o raciocínio matemático e a comunicação matemática.
\end{abstract}

Portanto, é perceptível a diferença dessa metodologia de ensino para o ensino tradicional, uma vez que o ensino exploratório permite ao aluno investigar os conhecimentos matemáticos presentes em um problema gerador, em que instigue os alunos a formularem ideias e levantar estratégias para a resolução, tal que o docente esteja preparado para os mais variados cenários que possam surgir durante o debate entre os alunos e o mediador, para esta situação necessita-se que o professor busque antecipadamente em seu planejamento, solucionar a tarefa das mais formas possíveis. Dessa maneira, o professor ao iniciar a aula aplicando esta tarefa incentiva os discentes a buscar uma resolução realizando questionamentos que os levam a pensar e explorar, conduzindo-os assim a uma aprendizagem significativa construída pelos seus próprios caminhos.

Diante disto, mostra-se necessário a função do professor como um mediador do conhecimento e como um participante ativo do ensino-aprendizagem dos alunos, de tal forma que indague as decisões e conclusões dos discentes durante o processo de resolução da tarefa, mas para que isso ocorra com maior eficácia é imposto um papel criterioso ao docente, o de buscar uma tarefa que vise atingir as metas de aprendizagem estabelecidas, a compreensão e o entendimento dos conteúdos que norteiam os possíveis caminhos para chegar a resolução. 
Contudo, o docente no momento de escolha da tarefa deve seguir os documentos educacionais brasileiros, bem como as orientações da instituição de ensino à qual é vinculado, de forma complementar, possui também a possibilidade de pesquisar em outras fontes seguras, materiais que auxiliem na compreensão dos objetivos que o conteúdo trabalhado abrange. Tais documentos orientam os assuntos que devem ser trabalhados na educação básica, dando ênfase à Base Nacional Comum Curricular BNCC (BRASIL, 2018), e os Parâmetros Curriculares Nacionais - PCN (2000).

De acordo com a BNCC, “[...] os estudantes devem desenvolver habilidades relativas aos processos de investigação, de construção de modelos e de resolução de problemas.” (BRASIL, 2018, p. 519), que estimulem o raciocínio crítico, representações e formulação de argumentos, de modo que ao concluir a resolução do problema matemático, possam apresentar o seu método utilizado, bem como interpretar as resoluções dos demais colegas, observando as semelhanças e diferenças entre elas. Assim, com o ensino exploratório, os alunos estarão inseridos em um círculo que foge do ensino tradicional e que estimule a participação dos discentes em discussões, em que eles possam expor seus pensamentos e ideias sobre o tema trabalhado, respeitando a opinião do próximo e construindo a própria aprendizagem, além de gerar um olhar crítico sobre futuras situações semelhantes.

De forma complementar à citação anterior, os PCN relatam em uma de suas finalidades que deve-se "[...]desenvolver as capacidades de raciocínio e resolução de problemas, de comunicação, bem como o espírito crítico e criativo[...]" (2000, p.49). Conforme a investigação e compreensão, os Parâmetros Curriculares Nacionais (2000, p.49) estabelecem objetivos gerais de matemática para o ensino, em que direcione o aluno a:

- Identificar o problema (compreender enunciados, formular questões etc).

- Procurar, selecionar e interpretar informações relativas ao problema.

- Formular hipóteses e prever resultados.

- Selecionar estratégias de resolução de problemas.

- Interpretar e criticar resultados numa situação concreta.

- Distinguir e utilizar raciocínios dedutivos e indutivos. 
- Fazer e validar conjecturas, experimentando, recorrendo a modelos, esboços, fatos conhecidos, relações e propriedades.

- Discutir ideias e produzir argumentos convincentes.

Como apresentado por estes documentos educacionais brasileiros, a BNCC e os PCN, o Ensino Exploratório relaciona-se diretamente com os objetivos destacados, com o intuito de inserir o aluno nesse cenário de indagações e questionamentos que impliquem em uma atuação direta no processo de resolução, sem a necessidade de realizar associações prévias ao conteúdo matemático abordado.

Portanto, o Ensino Exploratório de Matemática é uma metodologia de ensino que busca transformar e ressignificar a maneira de ensinar, de forma que a participação ativa do discente é fundamental para a construção do conhecimento, induzindo-o ao papel de protagonista no desenvolvimento de sua própria aprendizagem. Assim mantém-se um ritmo próprio no processo de compreensão dos conteúdos, nivelando o processo de aprendizagem.

\section{Tecnologias digitais como ferramentas no ensino remoto de matemática}

O acelerado desenvolvimento tecnológico e científico, propiciou a integração das potencialidades de recursos que resultaram na Internet, uma estrutura global que interliga os computadores e outros equipamentos para possibilitar o registro, produção, transmissão e recepção de informações e a comunicação entre indivíduos independentemente da posição geográfica (CAPOBIANCO, 2010).

Assim, ferramentas tradicionais no processo de ensino, como o quadro branco, vem perdendo a atração com o passar dos anos, e novas ferramentas estão sendo utilizadas para que se tenha a atenção dos alunos, dessa forma se faz necessário o uso de tecnologias para o ensino. Mas, o que são essas tecnologias? Adianta-se que tecnologias são bastante abrangentes, segundo Moran (2003):

Tecnologias são os meios, os apoios, as ferramentas que utilizamos para que os alunos aprendam. A forma como os organizamos em grupos, em salas, em outros espaços, isso também é tecnologia. O giz que escreve na lousa é tecnologia de comunicação e uma boa organização da escrita facilita e muito a aprendizagem. A forma de olhar, de gesticular, de falar com os outros, isso também é tecnologia. (MORAN, 2003, p. 153) 
Logo, na aula de matemática o uso de tecnologias como calculadoras, esquadros, compassos, réguas, entre outras, vem se tornando cada vez mais comum, mas quando fala-se em tecnologias digitais na aprendizagem, pensa-se primeiramente na internet, uma importante ferramenta que está presente no cotidiano da maioria das pessoas e que pode ser usada em benefício da aprendizagem. Além disso ainda é possível ter acesso a vários outros softwares que auxiliam nesse processo, Maltempi (2008) diz que:

\begin{abstract}
A sociedade impõe o uso da tecnologia na educação porque grande parte da população está em um crescente contato com ela no seu dia-a-dia. Dessa forma, cada vez mais as escolas recebem alunos usuários de tecnologias, habituados a elas, os quais naturalmente pressionam pelo seu uso na educação ao trazerem tecnologias para a sala de aula ou ao relacionarem as atividades realizadas na escola com a possibilidade de serem elaboradas com o apoio de tecnologias. (MALTEMPI, 2008, p.62)
\end{abstract}

Certamente grande parte da população usufrui da tecnologia, mas será que a utilizam de uma forma que agreguem conhecimentos e que desenvolvam habilidades para a vida pessoal, estudantil e/ou profissional? E quando aplicadas em sala de aula, possuem procedimentos metodológicos ou apenas são aplicadas? Esses são alguns questionamentos a serem feitos antes de se utilizar as tecnologias digitais no ambiente de ensino.

Em tempos de pandemia, o que era ensino presencial se tornou ensino remoto, ou seja, uma solução temporária para que as atividades pedagógicas pudessem continuar. Tendo a internet como ferramenta principal, as aulas online dão-se por meio de softwares, que podem ser baixados facilmente nos aparelhos disponíveis dos estudantes que possuem recursos tecnológicos para acompanhar as aulas, neste caso as aulas de matemática. Dessa forma, o uso das tecnologias digitais se faz necessário para a manutenção das práticas educacionais, deixando de ser uma alternativa, inovação, e passando a ser o principal meio para o desenvolvimento das atividades letivas, como por exemplo o uso do software geogebra no ensino de funções, uma importante ferramenta na área da geometria e álgebra em que auxilia na demonstração e na resolução de problemas nesse ambiente virtual, de forma que o estudante perceba com mais facilidade o seu erro (caso erre), assim instigando-o a procurar novos caminhos para se obter uma resolução. 


\section{A experiência da elaboração de uma aula utilizando o ensino exploratório da matemática durante o ensino remoto}

A disciplina de matemática é dita por muitos alunos como uma disciplina de difícil compreensão de seus conteúdos, dessa forma os professores tiveram que, em meio às dificuldades, buscar formas de proporcioná-los uma aprendizagem significativa. Várias ferramentas foram sendo testadas, slides prontos, slides a serem complementados diante a participação dos alunos, em tentativas de lousas online por exemplo, porém há conteúdos que exigem uma forma mais clara de representação e que o quadro branco faz falta durante a aula. Assim tem-se buscado sempre aperfeiçoar com o intuito de melhorar a qualidade das apresentações, procurando nas tecnologias digitais um suporte para um bom desenvolvimento das aulas remotas.

A aplicação da metodologia de ensino que tem sido citada ao longo deste artigo e que se deu utilizando tecnologias digitais, foi desenvolvida por meio de uma regência, em que foi trabalhado o conteúdo Sistema de Coordenadas no Plano Cartesiano na E.E.M.T.I. Adauto Bezerra, com os alunos do $1^{\circ}$ ano A. Para a realização da aula foi criada uma sala na plataforma Google Meet e enviado o link no grupo de Whatsapp da turma, esperamos uma quantidade considerável de alunos entrarem para que pudéssemos iniciar. Dando sequência, foi feita uma breve apresentação sobre o Programa Residência Pedagógica, e qual nosso objetivo com a regência. Antes de iniciar com o planejado realizamos alguns questionamentos aos estudantes, como por exemplo: se tinham conhecimento sobre localização de pontos, sentido de direção e locomoção no plano cartesiano por meio de passos estabelecidos, essas indagações foram feitas com o intuito de saber se os discentes já possuíam conhecimentos prévios sobre o assunto. A maioria entre dezenove alunos presentes na aula responderam que não tinham conhecimento sobre o tema e cerca de cinco deles disseram ter uma ideia de localização, porém ninguém possuía domínio dos conceitos necessários para a compreensão do conteúdo estabelecidos durante a criação do plano de aula, como por exemplo retas numéricas, abscissa, ordenada, quadrantes, pares ordenados e localizações no plano.

Em seguida apresentamos a tarefa introdutória da aula, apresentada abaixo, que foi retirado de um material estruturado de matemática disponibilizado pelo Sistema Online de Avaliação, Suporte e Acompanhamento Educacional (SISEDU), sendo esta 
O ensino exploratório como metodologia de ensino no processo de aprendizagem matemática por meio do ensino remoto

adaptada para que houvesse um maior aproveitamento dos conceitos matemáticos, além de trabalhar a interdisciplinaridade. Abaixo temos a tarefa aplicada:

- (SISEDU - ADAPTADO) O treinador Josias coletou dados sobre oito dos seus jogadores de basquete. Os pontos marcados no plano cartesiano mostram o número de minutos em quadra e a quantidade de pontos marcados na última partida pelos oito jogadores.

Imagem 2 - Plano cartesiano (minutos em quadra, pontos)

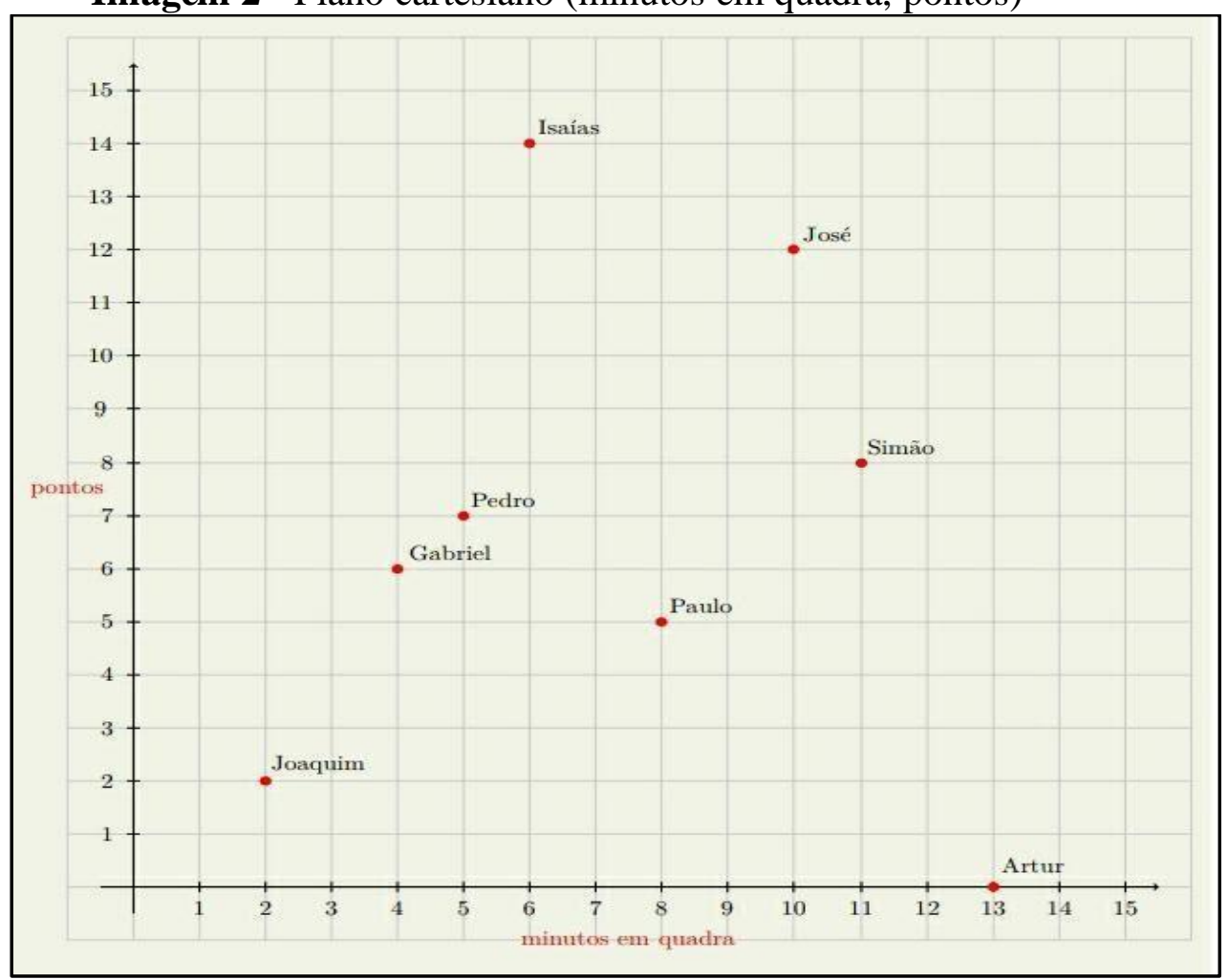

Fonte: Bessa; Neto (p.20).

- (a) Quais os três jogadores que tiveram melhor desempenho?

- (b) Analisando o plano cartesiano, se o treinador Josias quiser formar uma equipe inicial para a próxima partida com os jogadores que tiveram maior desempenho nesta partida, quais seriam esses jogadores?

- (c) Qual a quantidade de pontos realizados por essa equipe na última partida?

A tarefa foi escolhida e adaptada de forma que pudessem ser realizados diversos questionamentos, influenciando os alunos a desenvolverem um raciocínio matemático acerca do assunto, fazendo-os pensar, bem como utilizar de seus conhecimentos prévios para chegar a uma solução. Abaixo temos as resoluções mais comuns que esperávamos que os alunos apresentassem, e que de fato foi a única forma apresentada por eles: 


\section{RESPOSTAS:}

- Item A: Em $1^{\circ}$ lugar: Isaías, $2^{\circ}$ : Gabriel e em $3^{\circ}$ : Pedro, logo esses foram os três jogadores que apresentaram melhor desempenho nesta partida.

- Item B: Uma equipe de basquete é formada por cinco integrantes. A equipe que o treinador Josias iria selecionar para iniciar a próxima partida seria formada por: Isaías, Gabriel, Pedro, José e Joaquim, pois foram os cinco jogadores com melhor desempenho nesta partida.

- Item C: Cinquenta e quatro (54) pontos.

\section{RESOLUÇÕES:}

- ITEM A: Primeiro calcula-se a média de cada jogador na partida.

- $\quad$ Artur $=\frac{0}{13}=0,00$ pont. $/ \mathrm{min}$.

- Gabriel $=\frac{6}{4}=1,50$ pont. $/ \mathrm{min}$.

- Isaías $=\frac{14}{6}=2,33$ pont. $/ \mathrm{min}$.

- Joaquim $=\frac{2}{2}=1,00$ pont. $/ \mathrm{min}$.

- José $=\frac{12}{10}=1,20$ pont. $/ \mathrm{min}$.

- $\quad$ Paulo $=\frac{5}{8}=0,62$ pont. $/ \mathrm{min}$.

- $\quad$ Pedro $=\frac{7}{5}=1,40$ pont. $/ \mathrm{min}$.

- $\quad$ Simão $=\frac{8}{11}=0,72$ pont. $/ \mathrm{min}$.

- A partir dessa média, podemos afirmar quem foram os três jogadores com maior desempenho na partida, que são: Em $1^{\circ}$ lugar: Isaías, $2^{\circ}$ : Gabriel e em $3^{\circ}$ : Pedro.

- ITEM B: Ao analisar as médias do item anterior, podemos deduzir quem serão os titulares da próxima partida.

* Conhecimento interdisciplinar: O jogo de basquete é formado por dez (10) jogadores, sendo cinco (5) jogadores de cada equipe.

- Assim, com os dados do item anterior, podemos concluir que a equipe inicial da próxima partida seria formada por: Isaías, Gabriel, Pedro, José e Joaquim.

- ITEM C: De acordo com o plano cartesiano, temos a quantidade de pontos que cada jogador pontuou nesta rodada. 
- Artur fez 00 pontos; Gabriel fez 06 pontos; Isaías fez 14 pontos; Joaquim fez 02 pontos; José fez 12 pontos; Paulo fez 05 pontos; Pedro fez 07 pontos; Simão fez 08 pontos.

- Somando a quantidade de pontos, temos ao total:

- $(0+6+14+2+12+5+7+8)=54$ pontos.

Após debater a tarefa e os questionamentos que surgiram durante a discussão, alguns alunos apresentaram seus raciocínios para os demais, de forma que todos pudessem compreender como chegou-se a resolução. Logo em seguida reforçamos para eles o passo a passo deste conhecimento desenvolvido coletivamente pela turma, finalizando assim o processo de exploração da tarefa.

Por conseguinte, apresentamos aos discentes os conceitos básicos para o entendimento do assunto abordado, para isso utilizamos o software geogebra posicionando os jogadores no plano cartesiano e, em seguida explicamos sobre os pares ordenados, em que um par ordenado $(\mathrm{x}, \mathrm{y})$ encontra-se em uma localização diferente do par ordenado $(\mathrm{y}, \mathrm{x})$ quando $\mathrm{x}$ é diferente de $\mathrm{y}$, por exemplo.

\section{Considerações finais}

Com a finalização do presente artigo, concluiu-se que perante o ensino remoto, considerado um novo cenário em que a educação está inserida, a metodologia de Ensino Exploratório de matemática apresentou um resultado satisfatório, pois por meio de um questionário realizado de forma oral durante a aula, treze dos quinze alunos que responderam, afirmaram ter compreendido o tema da aula sobre sistemas de coordenadas no plano cartesiano, assim para sabermos a veracidade da resposta, questionamos a localização de coordenadas. Além disso relataram que a metodologia utilizada facilitou este acontecimento, visto que a mesma se torna uma precursora do diálogo/debate entre os estudantes, juntamente com o professor.

Com a aplicação dessa metodologia de ensino por meio do uso das ferramentas digitais, a aula tornou-se dinâmica de forma que os alunos se sentiram motivados a desenvolverem linhas de raciocínios matemáticos, tanto individualmente quanto coletivamente, além de ter sido possível obter um bom nível de participação dos estudantes, uma prática não tão comum como em aulas presenciais. Portanto, o debate 
surtido proporcionou aos alunos atuarem de maneira ativa na resolução da tarefa, assumindo um papel importante na busca por uma aprendizagem significativa. Dessa forma os discentes tornaram-se protagonistas na construção do seu próprio conhecimento matemático, visto que eles desenvolveram um pensamento crítico perante o que lhes foi apresentado, enquanto os mediadores atuaram fazendo conexões entre conhecimento e aluno.

\section{Referências}

BRASIL. Ministério da Educação. Secretaria de Educação Básica. Base Nacional Comum Curricular. Brasília: MEC, 2018.

BRASIL. Ministério da Educação. Secretaria de Educação Média e Tecnológica. Parâmetros Curriculares Nacionais (Ensino Médio). Brasília: MEC, 2000.

CANAVARRO, Ana Paula. Ensino Exploratório da matemática: Práticas e desafios. Educação e matemática. Ed. Associação de Professores de Matemática. Dezembro, 2011.

CAPOBIANCO, L. A. Revolução em curso: internet, sociedade da informação e cibercultura. Universidade de São Paulo: São Paulo, 2010.

MALTEMPI, Marcus Vinicius. Educação matemática e tecnologias digitais: reflexões sobre prática e formação docente. ACTA SCIENTIAE. 2008. Disponível em: Educação matemática e tecnologias digitais: reflexões sobre prática e formação docente / Mathematics education and digital technologies: Reflexions about the practice in teacher education | Maltempi | Acta Scientiae (ulbra.br). Acesso em 03 de fev. 2021.

MORAN, J.M. (2003) Gestão Inovadora com Tecnologias. In: Gestão Educacional e Tecnologia. VIEIRA, ALMEIDA E ALONSO, Alexandre Thomaz, Maria Elisabeth Bianconcini, Myrtes (org). São Paulo: Avercamp.

NETO, A. P.; BESSA, M. Raciocínio Geométrico: Coordenadas no Plano. Equipe Cientista Chefe. Sistema Online de Avaliação, Suporte e Acompanhamento Educacional-SISEDU. Ceará.

PONTE, J. P. (2005). Gestão curricular em Matemática. In GTI (Ed.), O professor e o desenvolvimento curricular (pp. 11-34). Lisboa: APM.

Recebido em: 06 / 03 / 2021

Aprovado em: 23 / 04 / 2021 